\title{
Incidence and Predictors of Cerebral Salt Wasting \\ Syndrome in Stroke Patients - Results of a Prospective Observational Study
}

Vindya Shalini Ranasinghe ( $\nabla$ vindyas@ahs.pdn.ac.lk)

University of Peradeniya

\section{Gayan Bowatte}

University of Peradeniya

Charles Antonypillai

National Hospital Kandy

Indika Bandara Gawarammana

University of Peradeniya

\section{Research Article}

Keywords: cerebral salt wasting syndrome, hyponatremia, stroke, syndrome of inappropriate antidiuretic syndrome

Posted Date: January 10th, 2022

DOI: https://doi.org/10.21203/rs.3.rs-1211512/v1

License: (9) This work is licensed under a Creative Commons Attribution 4.0 International License. Read Full License 


\section{Abstract}

\section{Background}

Cerebral salt wasting syndrome (CSWS) and Syndrome of Inappropriate Anti Diuretic Hormone secretion (SIADH) are the most common aetiological factors for developing hyponatremia following stroke. The differentiation of these two entities is crucial as the treatment options are completely different. Hence the knowledge on predictors of CSWS is important to make a more accurate diagnosis of CSWS.

\section{Methods}

Two hundred and fourty six patients with confirmed stroke were prospectively observed throughout the hospital stay in a tertiary referral center in Sri Lanka to identify the possible predictors of CSWS. Hyponatremia was defined as serum $\mathrm{Na}^{+}$level less than $131 \mathrm{mmo} / \mathrm{l}$. Serum osmolality, urine osmolality, urinary $\mathrm{Na}^{+}$, serum cortisol and thyroid function tests were performed on all the hyponatremic patients. Differentiation of the CSWS and SIADH was based on physical examination findings and laboratory parameters.

\section{Results}

The incidence of hyponatremia in our study population was 19.1\% (95\% Confidence Interval 14.39-24.58). The majority of patients $(24,51 \%)$ were attributed to CSWS. SIADH group comprised of $17(36.2 \%)$ patients and $6(12.7 \%)$ patients had other undetermined causes. There was a significant statistical difference between the aetiologies of hyponatremia and laboratory investigations like urinary $\mathrm{Na}^{+}$, urinary osmolality and serum osmolality. Demographic characteristics, comorbidities, imaging findings and clinical parameters like systolic blood pressure, diastolic blood pressure, on admission GCS were considered in the multivariable logistic regression model and the overall model was not significant.

\section{Conclusion}

The incidence of CSWS is higher than the incidence of SIADH. The demographic characteristics, comorbidities, imaging and clinical parameters like blood pressure, on admission GCS could not predict the occurrence of CSWS

\section{Background}

Hyponatremia is a common and important electrolyte abnormality seen in critically ill neurosurgical patients. Even though there are many aetiologies for hyponatremia, Syndrome of Inappropriate Anti Diuretic Hormone secretion (SIADH) and Cerebral Salt Wasting Syndrome (CSWS) are the main aetiological factors in neurological patients (1).

In SIADH, inappropriate release of vasopressin occurs which results in dilutional hyponatremia. In contrast, primary natriuresis along with depletion of extravascular volume is seen in CSWS (2). Reduced 
sympathetic neural input to the kidney and natriuretic peptide theory are two suggested theories for the occurrence of CSWS (3).

As both disorders can occur in intracranial pathologies and share same clinical and biochemical parameters, it is difficult to differentiate one from the other (4). The hallmark of differentiating these two entities lies in the extravascular volume status. In SIADH, the extravascular volume is normal or slightly high comparing to the low volume seen in CSWS. However the accurate determination of extravascular volume status is an absolutely a difficult task (5). Therefore, both clinical and biochemical data should be considered cumulatively to arrive at an accurate diagnosis of hypovolemia (6). Clinical signs of hypovolemia mainly include dry mucous membranes, increased skin turgor, sunken eyes and orthostatic hypotension. Biochemically there is elevated levels of hematocrit, s.albumin, blood urea nitrogen and s.creatinine values which indicate the presence of hypovolemia. In patients who are monitored in intensive care units, low pulmonary capillary pressure or low central venous pressure indicate the low circulating extravascular volume $(6,7)$. However assessing the serum levels of antidiuretic hormone or natriuretic peptides will not help to differentiate these two disorders (6).

Differentiating these two disorders is of utmost importance as they have different management approaches. Fluid restriction is indicated in uncomplicated SIADH. Isotonic saline is not recommended as it could further worsen the hyponatremia by retaining more water. The effect of using hypertonic saline is also not long lasting. Therefore, the use of diuretics and new class of drugs called vaptans are recommended in SIADH management (8).In contrast, replacing water is the first line management approach in CSWS. Isotonic saline is used and this would solve both hypovolemia and hyponatremia. If the condition is severe, hypertonic saline is recommended for the resuscitation (9). However, the correction of hyponatremia should be carried out with caution to prevent complications like osmotic demyelination syndrome (10). Previous literature provides evidence to suggest the importance of using mineralocorticoids in CSWS as they are potent in reabsorbing $\mathrm{Na}^{+}$form distal collecting tubules of the nephron (9).

The importance of differentiating these two disorders is obvious as they have totally different management approaches. Therefore it is important to study about parameters which could predict CSWS. Previous studies have investigated about the ability of haptoglobin gene and aneurysm location of sub arachnoid haemorrhage patients in predicting CSWS $(11,12)$. The purpose of this study is to investigate whether demographic data, past medical history, imaging parameters and some of the clinical parameters of stroke patients could predict the occurrence of CSWS.

\section{Methodology}

\subsection{Design and Setting}

A prospective cohort study was carried out on 246 stroke patients admitted to Teaching hospital Peradeniya located in the central province of Sri Lanka. The sample size calculation was based on the 
previously reported prevalence of hyponatremia of $20 \%$ due to intracerebral events(13). The following statistical formula was used to calculate the sample size.

$n=Z^{2} P(1-P) / d^{2}$

$\mathrm{n}=$ Sample Size

$Z=$ The statistic corresponding to level of confidence. This value is 1.96 if $a=5 \%$

$\mathrm{P}=$ Estimated prevalence of hyponatremia among stroke patients in hospital setting

$d=$ Maximum acceptable error $5 \%$

Ethical clearance to this study was obtained from the Ethics Review Committee, Faculty of Medicine, University of Peradeniya, Sri Lanka (2018/EC/44). Written informed consent was obtained from the patient or, in the instances where the patient was not clinically fit to do so, from the next of kin.

\subsection{Data collection}

The current paper is based on the data from patients of stroke recruited prospectively from 1st of June 2019 to 12th of February 2021. The diagnosis of stroke was established on the clinical history, examination and neuroimaging. Hyponatremia was defined as serum $\mathrm{Na}^{+}$level less than $131 \mathrm{mmo} / \mathrm{l}(14)$. Patients with known pre-event hyponatremia were excluded from the study. All the patients with low $\mathrm{Na}^{+}$ levels were assessed for their serum osmolality levels. The patients with low S. osmolality $(<285 \mathrm{mosm} / \mathrm{Kg})$ levels were further tested for their urine osmolality level. Those patients of high urine osmolality levels were assessed for their volume status. Moreover these patients were assessed for T4/TSH (Thyroid Stimulating Hormone) levels and S.Cortisol levels to exclude hypothyroidism and adrenal insufficiency respectively. The differentiation of the CSWS and SIADH was based on clinical examination findings, urinary $\mathrm{Na}^{+}$values and hematocrit values. CSWS was diagnosed if there was clinically declared dehydration, urinary $\mathrm{Na}^{+}$value $>50 \mathrm{mEq} / \mathrm{L}$ and elevated or normal hematocrit value. In contrast, SIADH was diagnosed if there was absence of clinical dehydration, urinary $\mathrm{Na}^{+}$value $20-$ $50 \mathrm{mEq} / \mathrm{L}$ and reduced or normal hematocrit value.

\subsection{Statistical analysis}

Descriptive statistics were calculated as means, standard deviations and percentages. Fisher's exact test or Pearson chi square test for categorical variables and Kruskal-Wallis test for continuous variables were used to compare the demographic, clinical, laboratory and imaging parameters with CSWS and SIADH groups. Incidence of hyponatremia and its $95 \%$ confidence intervals $(\mathrm{Cl})$ were calculated. Multivariate logistic regression analysis was performed to evaluate the predictors of CSWS. A P value of 0.05 or less than that is considered to be significant. The data analysis was done by using STATA version 16 (StataCorp. 2019. Stata Statistical Software:College Station, TX: StataCorp LLC.). 


\section{Results}

\subsection{Study participant characteristics}

The study sample comprised of 246 proven cases of stroke patients. The mean age of the study participants was 68.14 years (SD \pm 12.71 ). The percentage of male was $48.7 \%$ and the mean age was 68.25 years ( $S D \pm 12.25)$. The mean age of the females was 67.8 years ( $S D \pm 12.65)$ and there was no statistical difference between age of males and females $(P=0.778)$. Most of the patients $(57.32 \%)$ belonged to the age group of $60-79$ years. Among the study group, $27.2 \%$ were smokers and $64.3 \%$ were non-alcoholics.

In the study sample, $56.09 \%$ patients were on treatment for hypertension. However there was $21.1 \%$ patients who were not diagnosed previously, but having high blood pressure on admission and $28.8 \%$ of hypertensive patients had uncontrolled hypertension while on treatment. Of all the hypertensive patients, $52.5 \%$ were on lipid lowering drugs. Of the study sample, diabetes mellitus comprised of $30.8 \%$ of patients and $13 \%$ of patients were on lipid lowering drugs.

Of the 246 stroke patients, $79.7 \%$ had ischaemic stroke and $20.3 \%$ had haemorrhagic stroke. In the stroke patients' sample the prevalence of left sided stroke was $39.02 \%, 41.8 \%$ had right sided stroke and $19.1 \%$ had bilateral strokes.

\subsection{Evaluation of hyponatremia}

Out of 246 stroke patients, 47 patients (19.1\%) developed hyponatremia ( $95 \% \mathrm{Cl} 14.39-24.58)$. The majority of patients $(24,51 \%)$ were attributed to CSWS. There were 4 patients who had low hematocrit value $<37$ with urinary $\mathrm{Na}^{+}>100 \mathrm{mEq} / \mathrm{L}$ and those patients were considered to be CSWS as their renal salt wasting was significantly high, even though the hematocrit value was not suggestive of CSWS. SIADH group comprised of $17(36.2 \%)$ patients and $6(12.7 \%)$ patients had other undetermined causes. The mean day of development of hyponatremia was 1.81 days $(S D \pm 1.73)$ and there was no statistical difference between CSWS, SIADH and Other groups $(P=0.23)$. The stroke type and the difference in the levels of urinary $\mathrm{Na}^{+}$, serum osmolality and urine osmolality were statistically significant between each groups (Table 1). 
Table 1

The comparison of the aetiologies of hyponatremia and the demographic, clinical, imaging and laboratory parameters.

\begin{tabular}{|c|c|c|c|c|}
\hline Hyponatremic Patients (n-47) & $\begin{array}{l}\text { csWS } \\
(n-24)\end{array}$ & $\begin{array}{l}\text { SIADH } \\
(n-17)\end{array}$ & $\begin{array}{l}\text { Other } \\
(n-6)\end{array}$ & $P$ value \\
\hline \multicolumn{5}{|l|}{ Age } \\
\hline $40-59$ & $4(8.51 \%)$ & $1(2.13 \%)$ & 0 & 0.48 \\
\hline $60-79$ & $15(36.17 \%)$ & $11(23.4 \%)$ & $6(12.7 \%)$ & \\
\hline$>80$ & $5(10.64 \%)$ & $5(10.64 \%)$ & 0 & \\
\hline Gender & & & & 0.78 \\
\hline Male & $14(29.79 \%)$ & $8(17.02 \%)$ & $3(6.38 \%)$ & \\
\hline Female & $10(21.28 \%)$ & $9(19.15 \%)$ & $3(6.38 \%)$ & \\
\hline \multicolumn{5}{|l|}{ Stroke type } \\
\hline Ischaemic & $18(38.3 \%)$ & $16(34.04 \%)$ & $2(4.26 \%)$ & 0.01 \\
\hline Haemorrhagic & $6(12.77 \%)$ & $1(2.13 \%)$ & $4(8.51 \%)$ & \\
\hline \multicolumn{5}{|l|}{ Side of the stroke } \\
\hline Left & $13(27.66 \%)$ & $3(6.38 \%)$ & $2(4.26 \%)$ & 0.12 \\
\hline Right & $5(10.64 \%)$ & $9(19.15 \%)$ & $2(4.26 \%)$ & \\
\hline$B / L$ & $6(12.77 \%)$ & $5(10.64 \%)$ & $2(4.26 \%)$ & \\
\hline On admission Glasgow coma scale (GCS) & $\begin{array}{l}12.58 \\
(S D \pm 2.6)\end{array}$ & $\begin{array}{l}12.82 \\
(S D \pm 3.06)\end{array}$ & $\begin{array}{l}13.83 \\
(S D \pm 2.04)\end{array}$ & 0.56 \\
\hline Systolic blood pressure(mmHg) & $\begin{array}{l}151.83 \\
(S D \pm 31.37)\end{array}$ & $\begin{array}{l}151.29 \\
(S D \pm 32.14)\end{array}$ & $\begin{array}{l}166.17 \\
(S D \pm 36.27)\end{array}$ & 0.67 \\
\hline Diastolic blood pressure $(\mathrm{mmHg})$ & $\begin{array}{l}87.6 \\
(S D \pm 21.46)\end{array}$ & $\begin{array}{l}89.3 \\
(S D \pm 17.8)\end{array}$ & $\begin{array}{l}90.5 \\
(S D \pm 15.9)\end{array}$ & 0.77 \\
\hline \multicolumn{4}{|l|}{ Laboratory investigations } & Serum $\mathrm{Na}^{+}(\mathrm{mmol} / \mathrm{l})$ \\
\hline \multirow{2}{*}{ Urinary $\mathrm{Na}^{+}(\mathrm{mmol} / \mathrm{l})$} & $\begin{array}{l}130.6 \\
(S D \pm 3.05)\end{array}$ & $\begin{array}{l}129.36 \\
(\mathrm{SD} \pm 3.9)\end{array}$ & $\begin{array}{l}130.13 \\
(S D \pm 4)\end{array}$ & 0.58 \\
\hline & $\begin{array}{l}108.6 \\
(S D \pm 42.9)\end{array}$ & $\begin{array}{l}38.6 \\
(S D \pm 15.45)\end{array}$ & $\begin{array}{l}20.4 \\
(S D \pm 11.34)\end{array}$ & 0.0001 \\
\hline
\end{tabular}


Serum Osmolality (mosm/Kg)

Urine Osmolality

(mosm $/ \mathrm{Kg})$
278.5

$(\mathrm{SD} \pm 14.25)$

534.88

$(S D \pm 227.8)$
276.3

$(\mathrm{SD} \pm 12.94)$

349

$(\mathrm{SD} \pm 82.5)$

$(S D \pm 18.5)$

$(\mathrm{SD} \pm 74.1)$

${ }^{1}$ Kruskal-Wallis test and Fisher's exact test or Pearson chi square test were performed to get the $P$ value ${ }^{2}$ Bold indicates statistical significance

\subsection{Predictors of CSWS in stroke patients}

The multivariate logistic regression analysis of CSWS patients revealed that the overall model was not significant $\left\{P=0.12\right.$, pseudo $R^{2}=0.25$ with a log likelihood of -24.36 (Table 2$\left.)\right\}$.

Table 2

Multivariate logistic regression model for CSWS patients (n-47)

\begin{tabular}{|lllll|}
\hline & Odds Ratio & \multicolumn{2}{l|}{$95 \%$ confidence interval } & P value \\
\hline Age & 0.97 & 0.88 & 1.05 & 0.46 \\
Gender & 0.32 & 0.04 & 2.13 & 0.24 \\
Female & & & & \\
\hline Hypertension & 0.41 & 0.05 & 2.89 & 0.37 \\
\hline Dyslipidaemia & 4.4 & 0.41 & 47.8 & 0.22 \\
\hline Diabetes mellitus & 5.14 & 0.81 & 32.42 & 0.08 \\
\hline Systolic Blood pressure & 1 & 0.97 & 1.04 & 0.71 \\
\hline Diastolic blood pressure & 0.95 & 0.9 & 1.02 & 0.19 \\
\hline On admission GCS & 0.81 & 0.56 & 1.17 & 0.27 \\
\hline Stroke type & 1.54 & 0.21 & 11.19 & 0.66 \\
Haeamorrhagic & & & & \\
\hline Side of the stroke & 0.15 & 0.02 & 1.1 & 0.06 \\
\hline Right & & & & \\
\hline
\end{tabular}

In this group of patients, none of the factors that were considered in the model could predict the occurrence of CSWS in stroke patients.

\section{Discussion}


Of the 246 stroke patients, 47 developed hyponatremia. Majority of patients developed CSWS. SIADH group comprised of 17 patients and 6 patients had other undetermined causes. There was a significant statistical difference between the aetiologies of hyponatremia and laboratory investigations like urinary $\mathrm{Na}^{+}$, urinary osmolality and serum osmolality. Demographic characteristics, comorbidities, imaging and clinical parameters like blood pressure, on admission GCS were considered in the multivariate logistic regression model and none of the factors could predict the occurrence of CSWS.

The incidence of hyponatremia in our study group was $19.1 \%$. This finding is consistent with Natarajan et al study which reported incidence of hyponatremia as $20 \%$ (13).However there were studies which showed higher hyponatremia incidence as high as $45.3 \%$ (15). In our study there was higher incidence of CSWS (51\%). In contrast the SIADH group showed lower incidence of $36.2 \%$. These results are in consistent with the study done by Kalita et al where they showed $44.2 \%$ of CSWS cases and $7 \%$ of SIADH cases. Further they concluded that cerebral salt wasting syndrome as the most common aetiology of hyponatremia in stroke patients (16). However, there were studies which oppose these findings. One such study revealed that out of all the cases of hyponatremia, SIADH was found in $67 \%$ of patients and CSWS in $33 \%$ of patients (17). There was another interesting study done on hyponatremia in subarachnoid haemorrhage patients in which they have found no cases of CSWS (18).

There was a statistically significant difference in urinary $\mathrm{Na}^{+}$levels, S.osmolality and $\mathrm{U}$. osmolality between the aetiologies of hyponatremia. One previous study has shown a significant difference $(P=$ 0.001) in urinary $\mathrm{Na}^{+}$between SIADH and CSWS (19). However, most of the literature suggests that these parameters could not differentiate the aetiologies of hyponatremia and proper evaluation of the extracellular volume is mandatory (14).

According to our study, the demographic characteristics like age and gender, the comorbidities like hypertension, dyslipidemia and diabetes mellitus, the clinical parameters like systolic blood pressure, diastolic blood pressure and on admission GCS and the imaging data like type of stroke, side of the stroke could not predict the occurrence of CSWS. However, studies have been done to identify the possible predictors of CSWS following SAH previously. One such study has evaluated the importance of using haptoglobin gene as a genetic marker in predicting the CSWS. Haptoglobin gene is associated with the inflammation following SAH and some experts believe that CSWS occurs as a result of this inflammatory process. However the inflammatory process is different among the various groups of haptoglobin genotypes and it was concluded that HP2-2 genotype is an independent predictor of CSWS (12). In contrast, the aneurysm location and the spread of bleeding in SAH do not predict the development of CSWS $(11,20)$.

\section{Conclusion}

The incidence of CSWS is higher than the incidence of SIADH. The differentiation of these two entities is important as they have different management approaches. Therefore it is better to have factors which could predict the occurrence of CSWS. However according to our study demographic characteristics, 
comorbidities, imaging and clinical parameters like blood pressure, on admission GCS could not predict the occurrence of CSWS.

\section{Abbreviations}

CSWS - Cerebral Salt Wasting Syndrome

SIADH-Syndrome of Inappropriate Anti diuretic Hormone secretion

GCS- Glasgow Coma Scale

Cl- Confidence Interval

\section{Declarations}

1) Ethics approval and consent to participate

Ethical clearance to this study was obtained from the Ethics Review Committee, Faculty of Medicine, University of Peradeniya, Sri Lanka (2018/EC/44).

All methods were performed in accordance with the relevant guidelines and regulations.

Informed consent was obtained from the patient or, in the instance where the patient was not clinically fit to do so or who were dead, from the next of kin and legal guardians.

2) Consent for publication

Not applicable

3) Availability of data and materials

All data generated or analysed during this study are included in this published article.

4) Competing interests (financial and non-financial)

The authors have declared no competing interests exist

5) Funding

Funding for this research was provided by University grants of University of Peradeniya, Sri Lanka (Grant No: URG/2018/08/AHS)

6) Author contribution

Conceptualization: Vindya Shalini Ranasinghe, Indika Bandara Gawarammana 
Methodology: Vindya Shalini Ranasinghe, Indika Bandara Gawarammana, Charles Antonypillai

Formal analysis and investigation: Vindya Shalini Ranasinghe, Gayan Bowatte

Writing- original draft preparation: Vindya Shalini Ranasinghe

Writing- Review and editing: Vindya Shalini Ranasinghe, Indika Bandara Gawarammana, Gayan Bowatte, Charles Antonypillai

Resources: Vindya Shalini Ranasinghe, Indika Bandara Gawarammana

Supervision: Indika Bandara Gawarammana

7) Acknowledgement

The authors wish to thank the University of Peradeniya for providing funds and the staff of the medical wards, Teaching Hospital Peradeniya for their support in data collection process.

\section{References}

1. Tisdall M, Crocker M, Watkiss J. Europe PMC Funders Group Disturbances of sodium in critically ill adult neurologic patients: a clinical review. 2006;18(1):57-63.

2. Coenraad MJ, Meinders AE, Taal JC, Bolk JH. Hyponatremia in intracranial disorders. Neth J Med. 2001;58(3):123-7.

3. Palmer BF. Hyponatremia in patients with central nervous system disease: SIADH versus CSW. Trends Endocrinol Metab. 2003;14(4):182-7.

4. Yee AH, Burns JD, Wijdicks EFM. Cerebral Salt Wasting: Pathophysiology, Diagnosis, and Treatment. Neurosurg Clin N Am [Internet]. 2010;21(2):339-52. Available from: http://dx.doi.org/10.1016/j.nec.2009.10.011

5. Rabinstein AA, Wijdicks EFM. Hyponatremia in Critically III Neurological Patients. Neurologist. 2003;9(6):290-300.

6. Guerrero R, Pumar A, Soto A, Pomares MA, Palma S, Mangas MA, et al. Early hyponatraemia after pituitary surgery: Cerebral salt-wasting syndrome. Eur J Endocrinol. 2007;156(6):611-6.

7. Cui H, He G, Yang S, Lv Y, Jiang Z, Gang X, et al. Inappropriate Antidiuretic Hormone Secretion and Cerebral Salt-Wasting Syndromes in Neurological Patients. Front Neurosci. 2019;13(November):111.

8. Oh JY, Shin J II. Syndrome of inappropriate antidiuretic hormone secretion and cerebral/renal salt wasting syndrome: Similarities and differences. Front Pediatr. 2015;2(JAN):1-5.

9. Dholke H, Campos A, Reddy CNK, Panigrahi MK. Cerebral salt wasting syndrome. 2016;205-10.

10. Cerdà-Esteve M, Cuadrado-Godia E, Chillaron JJ, Pont-Sunyer C, Cucurella G, Fernández M, et al. Cerebral salt wasting syndrome: Review. Eur J Intern Med. 2008;19(4):249-54. 
11. Hoffman H, Ziechmann R, Gould G, Chin LS. The Impact of Aneurysm Location on Incidence and Etiology of Hyponatremia Following Subarachnoid Hemorrhage. World Neurosurg [Internet]. 2018;110:e621-6. Available from: https://doi.org/10.1016/j.wneu.2017.11.058

12. Murthy SB, Caplan J, Levy AP, Pradilla G, Moradiya Y, Schneider EB, et al. Haptoglobin 2-2 genotype is associated with cerebral salt wasting syndrome in aneurysmal subarachnoid hemorrhage. Neurosurgery. 2015;78(1):71-6.

13. Natarajan DK, Prasad DM. "Hyponatremia in Stroke: Cerebral Salt Wasting Versus Syndrome of Inappropriate Anti-Diuresis." IOSR J Dent Med Sci. 2016;15(07):01-22.

14. Rahman M, Friedman WA. Hyponatremia in neurosurgical patients: Clinical guidelines development. Neurosurgery. 2009;65(5):925-35.

15. Shah A, Sabir S, Artani M, Salam O, Khan S, Rizwan A. Significance of Hyponatremia as an Independent Factor in Predicting Short-term Mortality in Patients with Hemorrhagic Stroke. Cureus. 2019;11(4).

16. Kalita J, Singh RK, Misra UK. Cerebral Salt Wasting Is the Most Common Cause of Hyponatremia in Stroke. J Stroke Cerebrovasc Dis [Internet]. 2017;26(5):1026-32. Available from: http://dx.doi.org/10.1016/j.jstrokecerebrovasdis.2016.12.011

17. Saleem S, Yousuf I, Gul A, Gupta S, Verma S. Hyponatremia in stroke. Ann Indian Acad Neurol. 2014;17(1):55-7.

18. Hannon MJ, Behan LA, O’Brien MMC, Tormey W, Ball SG, Javadpur M, et al. Hyponatremia following mild/moderate subarachnoid hemorrhage is due to SIAD and glucocorticoid deficiency and not cerebral salt wasting. J Clin Endocrinol Metab. 2014;99(1):291-8.

19. Karunanandham S, Rajappa T, Selvaraju K. Hyponatremia in patients admitted with stroke. J Clin Diagnostic Res. 2018;12(8):0C34-6.

20. Kojima J, Katayama Y, Moro N, Kawai H, Yoneko M, Mori T. Cerebral salt wasting in subarachnoid hemorrhage rats: Model, mechanism, and tool. Life Sci. 2005;76(20):2361-70. 